\title{
Energy harvesting in the nonlinear electromagnetic system
}

\author{
K. Kucab ${ }^{a}$, G. Górski, and J. Mizia \\ Faculty of Mathematics and Natural Sciences, University of Rzeszów, ul. Pigonia 1, \\ 35-310 Rzeszów, Poland
}

Received 17 August 2015 / Received in final form 9 September 2015 Published online 20 November 2015

\begin{abstract}
We examine the electrical response of electromagnetic device working both in the linear and nonlinear domain. The harvester is consisted of small magnet moving in isolating tube surrounded by the coil attached to the electrical circuit. In the nonlinear case the magnet vibrates in between two fixed magnets attached to the both ends of the tube. Additionally we use two springs which limit the movement of the small magnet. The linear case is when the moving magnet is attached to the repelling springs, and the static magnets have been replaced by the non-magnetic material. The potentials and forces were calculated using both the analytical expressions and the finite elements method. We compare the results for energy harvesting obtained in these two cases. The generated output power in the linear case reaches the peak value $80 \mathrm{~mW}$ near the resonance frequency $\omega_{0}$ for maximum base acceleration considered by us, whereas in the non-linear case the corresponding outpot power has the peak value $95 \mathrm{~mW}$ and additionally relatively high values in the excitation frequencies range up to $\omega=1.2 \omega_{0}$. The numerical results also show that the power efficiency in the nonlinear case exceeds the corresponding efficiency in the linear case at relatively high values of base accelerations greater than $5 \mathrm{~g}$. The results show the increase of harvested energy in the broad band of excitation frequencies in the nonlinear case.
\end{abstract}

\section{Introduction}

Energy harvesting from small vibrations seems to be very promising topic in many electronic applications. The devices converting the mechanical energy, which in many cases is wasted, into the electrical energy seem to be very important. This kind of the devices can be used as the power supply for the sensors located in hard to reach places or in the case when it is difficult to supply the power to the device. They can also be used as the power source for small electronic devices which have low power consumption.

The analysis of energy harvesting device has to take into account the fact, that the generated power should be relatively high and in addition to be constant in the broad

\footnotetext{
${ }^{a}$ e-mail: kkucab@ur.edu.pl
} 


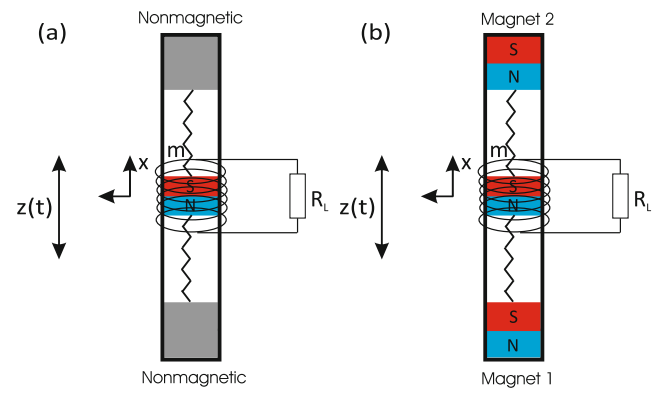

Fig. 1. Schematic of the device considered in this work, (a) the linear case, (b) the nonlinear case.

frequency band. In practice, the harvesting device should generate high output power in the broad range of frequencies, which makes it useful in many applications. There are many papers devoted to this subject. Great number of publications take into account the utilization of piezoelectric effect. The energy harvesting devices based on this effect use small magnets attached to the piezoelectric beams moving in magnetic field generated by the static magnets [1-9]. The nonlinearity of the potential energy is used to achieve the extended frequency bandwidth of the harvester. There are also many papers devoted to the energy harvesting devices based on electromotive force phenomena [10-12]. In these papers the generated voltage is produced by the coil inside which vibrates the small magnet. The quasi-periodic movement of this magnet can be achieved by the magnetic field generated by fixed magnets or additionally by the springs attached to the moving magnet and the static ones. Lee et al. [13] examined (theoretically and experimentally) the electromagnetic system similar to our device. In the nonlinear case they used the three magnet device, where the center magnet levitated between fixed ones. In our work we use opposite magnet polarizations. The authors also used in the model approximate expression (fifth order fit) for the dependence of magnetic force vs. displacement between magnes, while in our work we used the integral relation. The authors analyzed the frequency response of the linear and nonlinear system. They have shown that the nonlinear harvester has significantly higher bandwidth compared to the linear one. Mann and Sims [14] also examined the harvesting device that uses magnetic levitation. This model is examined for the case of harmonic base excitation. The authors state that "engaging the nonlinear respondse of the system can result in relatively large oscillations over a wider range of frequencies". In this paper we use the electromagnetic method to generate electrical power. The device works in the nonlinear domain caused by the influence of both the magnetic field and Hook's potential of the springs.

\section{The model}

The device considered by us is consisted of small magnet moving in the magnetic field generated by the two fixed magnets attached directly to the ends of isolating tube which restricts the harvester's motion. Friction effects were neglected for simplicity. The tube is surrounded by the coil attached to the electric load on which the output power is generated. The schematic diagram of the considered device is presented in Fig. 1. We consider two cases. First is the case of the moving magnet oscillating between two nonmagnetic bodies (Fig. 1a). The device consists additionally of two springs which are attached to the moving magnet and the static body. The second case is when the static bodies are magnetic (Fig. 1b). 


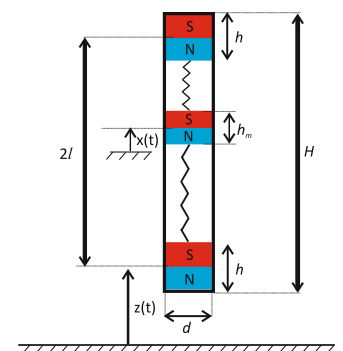

Fig. 2. Dimensions and notation used for the considered device.

In the first case the potential energy is quadratic and the restoring force is linear with displacement. In the second case the magnet is moving between static attracting magnets with added springs between them. The potential energy is relatively flat in large interval of displacement with some side minima. The depth of these minima depends on relative strength of springs and attracting magnets.

The moving magnet is characterized by its mass $m$. The static magnets and the springs cause the moving magnet to "feels" the potential arising from the magnetic field and from the Hooke's law. The moving magnet is surrounded by the coil consisted of $N=1300$ turns and $6.5 \mathrm{~cm}$ length. The coil is wound on the tube. The change of magnetic flux covered by the coil generates the output voltage and the electric power on the load $R_{L}$ connected to this coil. Due to relatively small mass of the moving magnet the gravitational effects can be neglected. Additionally, the device can work both in the vertical and horizontal direction, depending on the direction of harmonic excitation, $z(t)$, applied to the harvester. The displacements and dimensions of the magnets for analyzed device are presented in Fig. 2.

The dynamics of the moving magnet is described in terms of the differential equations which couples its relative displacement $y(t)=x(t)-z(t)$, where $x(t)$ is the displacement of the moving magnet with respect to its equilibrium position, with the current $I(t)$ flowing through the electric load. We use the Newton's law

$$
-\ddot{z}(t)=\ddot{y}(t)+\frac{1}{m}\left[c \dot{y}(t)-k y(t)-\chi I(t)+F_{\operatorname{magn}}(y)\right],
$$

with the equation for electrical current $I(t)$ obtained from the Kirchhoff law

$$
L \dot{I}(t)+R I(t)-\chi \dot{y}(t)=0 .
$$

The over-dot sign means the time derivative, and the resistance $R$ is the sum of internal and external resistive load part, $R=R_{L}+R_{\text {int }}$. In Eqs. (1) and (2) $m$ is the moving magnet's mass, $k=2 k_{0}$, where $k_{0}$ is the stiffness, $c$ is the coefficient related to the mechanical energy dissipation, $\chi$ is related to the transducer properties, and $L$ is the inductance of the coil. The transducer property $\chi$ depends on a position of magnets against coil. One should remember, that this property (in this arrangement) does not have a constant value. Taking into account the Faraday's law, the magnetic flux density is proportional with magnet position and coil volume.

The interaction between magnets creates the force term, $F_{\text {magn }}$, which is described by the following equation (see [15])

$$
F_{\text {magn }}(y)=I_{1}(y)-I_{2}(y),
$$

where

$$
I_{1}(y)=\pi \mu_{0} M^{2} d^{2} \int_{0}^{\infty} \frac{J_{1}^{2}(q)}{q} \sinh \left(q \frac{h}{d}\right) \sinh \left(q \frac{h_{m}}{d}\right) e^{-q \frac{\left(2 y+h+h_{m}\right)}{d}} d q,
$$


Table 1. The parameters of the magnets used in computations. $M$ is the magnetization, $d$ - diameter, $h$ - height. Subscript $m$ denotes the moving magnet.

\begin{tabular}{lll}
\hline Variable name & Variable value & Variable unit \\
\hline$M$ & 925550 & {$[\mathrm{~A} / \mathrm{m}]$} \\
$H$ & 85 & {$[\mathrm{~mm}]$} \\
$d$ & 20 & {$[\mathrm{~mm}]$} \\
$h$ & 10 & {$[\mathrm{~mm}]$} \\
$h_{m}$ & 5 & {$[\mathrm{~mm}]$} \\
\hline
\end{tabular}

$$
I_{2}(y)=\pi \mu_{0} M^{2} d^{2} \int_{0}^{\infty} \frac{J_{1}^{2}(q)}{q} \sinh \left(q \frac{h}{d}\right) \sinh \left(q \frac{h_{m}}{d}\right) e^{-q \frac{\left(4 l-h-h_{m}-y\right)}{d}} d q .
$$

In Eqs. (4) and (5) $M$ is the magnetization of the magnet (for simplicity $M$ is assumed the same for all magnets), and $J_{1}$ is the modified Bessel function of the first kind. In our computations we will use the properties of neodymium iron boron 36/26 permanent magnets with $M=925550[\mathrm{~A} / \mathrm{m}]$ (at the temperature $T=20^{\circ} \mathrm{C}$ ). It is also assumed that the base acceleration has the following form

$$
\ddot{z}(t)=A_{0} \sin \omega t,
$$

where $A_{0}$ is the base acceleration amplitude. We use the standard relation connecting an angular frequency $\omega$ with the applied frequency $f: \omega=2 \pi f$. To solve the set of coupled equations (1) and (2) we transform it to the dimensionless form:

$$
\begin{gathered}
y^{\prime \prime}(\tau)+\tilde{c} y^{\prime}(\tau)-y(\tau)-\tilde{I}(\tau)+\tilde{F}_{\operatorname{magn}}(y)=-\tilde{A}_{0} \sin (\tilde{\omega} \tau), \\
\tilde{I}^{\prime}(\tau)+\tilde{R} \tilde{I}(\tau)-\tilde{\alpha} y^{\prime}(\tau)=0,
\end{gathered}
$$

where the prime sign denotes the differentiation with respect to dimensionless time defined as $\tau=\omega_{0} t$ which is related to the systems natural frequency $\omega_{0}=\sqrt{k / m}$ (without magnetic field). Additionally, we have used the following notations:

$$
\tilde{\omega}=\frac{\omega}{\omega_{0}}, \tilde{c}=\frac{\omega_{0} c}{k}, \tilde{\chi}=\frac{\chi}{k}, \tilde{R}=\frac{R}{\omega_{0} L}, \tilde{\alpha}=\frac{\chi^{2}}{k L}, \tilde{I}=\tilde{\chi} I, \tilde{F}_{\mathrm{magn}}=\frac{F_{\mathrm{magn}}}{k}, \tilde{A}_{0}=\frac{A_{0}}{\omega_{0}^{2}} .
$$

Permanent magnets used by us are Nd-Fe-B magnets, and they are characterized by the parameters presented in Table 1.

The other parameters describing the properties of the device examined by us are presented in Table 2 .

\section{Numerical results}

First, taking into account the relations (3)-(5) we calculate the magnetic force acting on the moving magnet moving between the static magnets. After adding up the harmonic force from springs this will allow to calculate later on the total force in the nonlinear case. We also calculate the force between static and moving magnets using Infolytica MagNet package. The results together with the analytical results are shown in Fig. 3. 
Table 2. The parameters used in computations. $R_{L}$ is the electrical load, $L$ - coil's inductance, $R_{\text {int }}$ - coil's internal resistance, $k$ - stiffness.

\begin{tabular}{lll}
\hline Variable name & Variable value & Variable unit \\
\hline$R_{L}$ & $10-100000$ & {$[\Omega]$} \\
$R_{\text {int }}$ & 787 & {$[\Omega]$} \\
$L$ & 12.5 & {$[\mathrm{mH}]$} \\
$k$ & 164.25 & {$[\mathrm{~N} / \mathrm{m}]$} \\
$c$ & $5.4 \cdot 10^{-3}$ & {$[\mathrm{~kg} / \mathrm{s}]$} \\
$\chi$ & 5 & {$[\mathrm{~N} / \mathrm{A}]$} \\
\hline
\end{tabular}

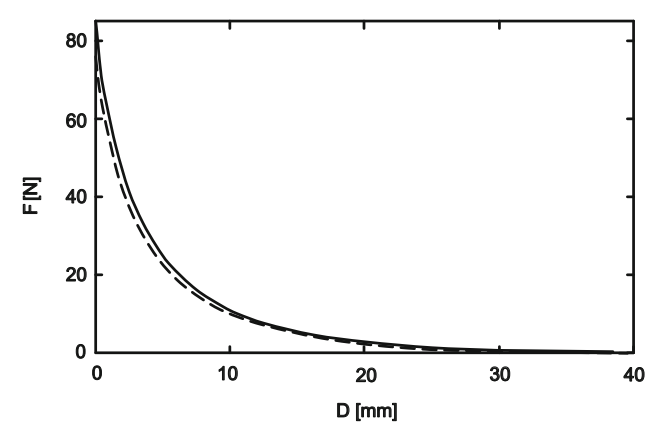

Fig. 3. Magnetic force as a function of distance $D$ between the static and moving magnet. The finite elements method results (Infolytica MagNet package) - solid line; the analytical solution - dashed line.

As we can see, the significant values of interacting force between moving and static magnet appear in the region of distance which is less than $3 \mathrm{~cm}$. Additionally, Fig. 3 shows that the analytical results are similar to the results of the finite element method (maximum error of the order of $6.5 \%$ appears in the case of the magnets contacting with each other). In the case of two attracting magnets touching themselves the exact result obtained by the simple calculation is given by the equation:

$$
F_{\text {magn }}=\frac{\bar{B}^{2} S}{2 \mu_{0}},
$$

where $S$ is the area (perpendicular to $\mathbf{B}$ ) of the magnet. Taking into account the value of $\mathbf{B}$ obtained from the Infolytica package (see Inset - Fig. 4 ) we have $F_{\text {magn }} \approx 82.3 \mathrm{~N}$, which is in a good agreement with both analytical and numerical finite elements result.

The above approximation for maximum force acting between magnets allows to estimate the proper parameters for the springs to obtain bistable potential. For the springs made of steel wire (diameter equal to $0.4 \mathrm{~mm}$ ) which consist of approximately 5.5 turns we assume $7 \mathrm{~mm}$ diameter. With these dimensions, both springs together can balance the maximum magnetic force between static and moving magnet when the magnets are separated by $1.5 \mathrm{~cm}$. Additionally, when the spring reaches its minimum length and its turns are packed closely it will be acting on a moving magnet as a stopper which restricts the minimum distance between magnets to the value of the order of $2.3 \mathrm{~mm}$.

Including the physical properties of the springs we can calculate the effective force acting on the moving magnet in the second case, i.e. when the magnet is moving between static magnets. The numerical results are presented in Fig. 5. 


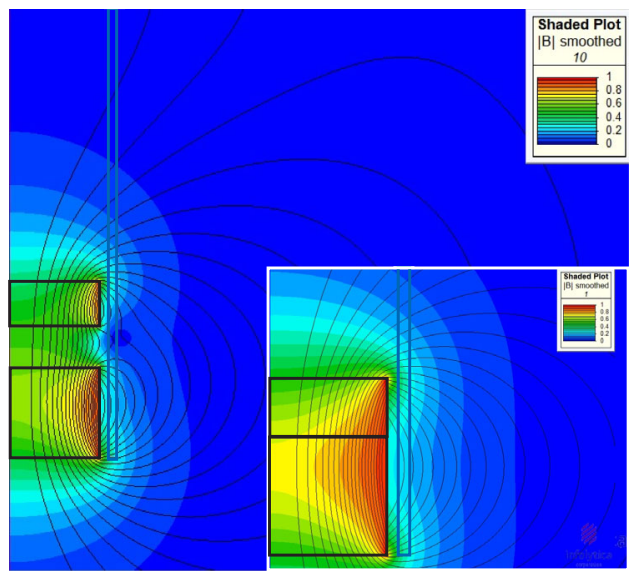

Fig. 4. Magnetic field (in $[\mathrm{T}]$ ) and the magnetic flux lines in the system of attracting magnets obtained from the finite elements method. Since the problem has an axial symmetry we show only the right half of the device. The bottom static magnet and the moving magnet $4.5 \mathrm{~mm}$ away from it is visible. Inset - the magnets are touching themselves (the color scale is the same as in main figure).

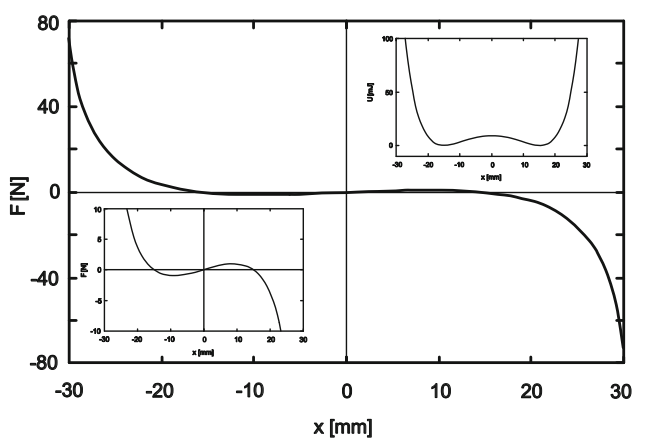

Fig. 5. Effective force between the static and moving magnets with springs. The negative values mean the force is opposite to the $x$ direction (see Fig. 2). Upper right inset - the potential energy of the moving magnet normalized to 0 in the equilibrium position.

After calculating the effective forces in both linear and nonlinear case, we can obtain the electrical power generated by harvesting device considered by us. We use the standard relation $\bar{P}=\left\langle I^{2}\right\rangle R_{L}$, where the $\left\langle I^{2}\right\rangle$ is the mean squared current flowing through the electric load $R_{L}$, which is chosen equal to $R_{L}=2 \mathrm{k} \Omega$. For this value of $R_{L}$ the effective output power reaches its maximum value (see Fig. 10).

In Fig. 6 we show the generated output power as a function of frequency (scaled to the resonance frequency which is $f \approx 26.6 \mathrm{~Hz}$ ) for different values of base acceleration amplitude in the linear case. We have to mention here that the base acceleration amplitudes used by us were chosen to give the review of system's response in wide range of accelerations. In real life the used levels of vibrations are very dangerous for human body, so our considerations are mainly theoretical.

As we can see, for low values of base acceleration amplitude, we have classical resonance peak near resonance frequency (see Fig. 6a, 6b). The width of the resonance peak increases, when the springs start to act on the moving magnet as the stoppers (excitation force is large enough to force magnet to compress the spring to its minimum width; see Fig. 6c, 6d). The "chaotic" shape of the curves is caused by the sensitivity of differential equations to initial conditions. 

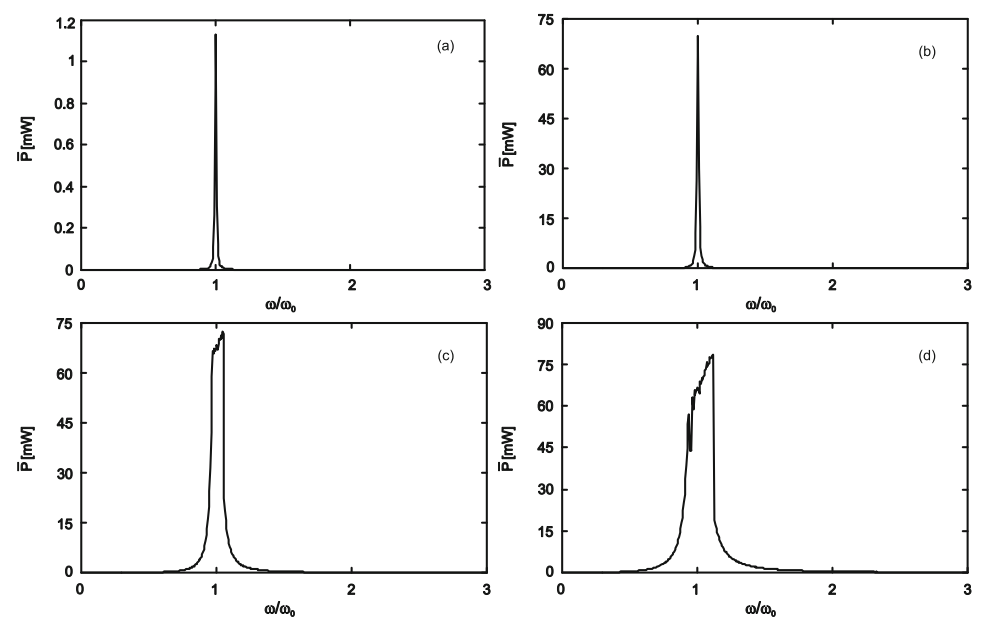

Fig. 6. Electric power vs. frequency of excitations in the linear case for different values of base acceleration amplitude: (a) $A_{0}=0.1 \mathrm{~g}$; (b) $A_{0}=1 \mathrm{~g}$; (c) $A_{0}=5 \mathrm{~g}$; (d) $A_{0}=10 \mathrm{~g}$.
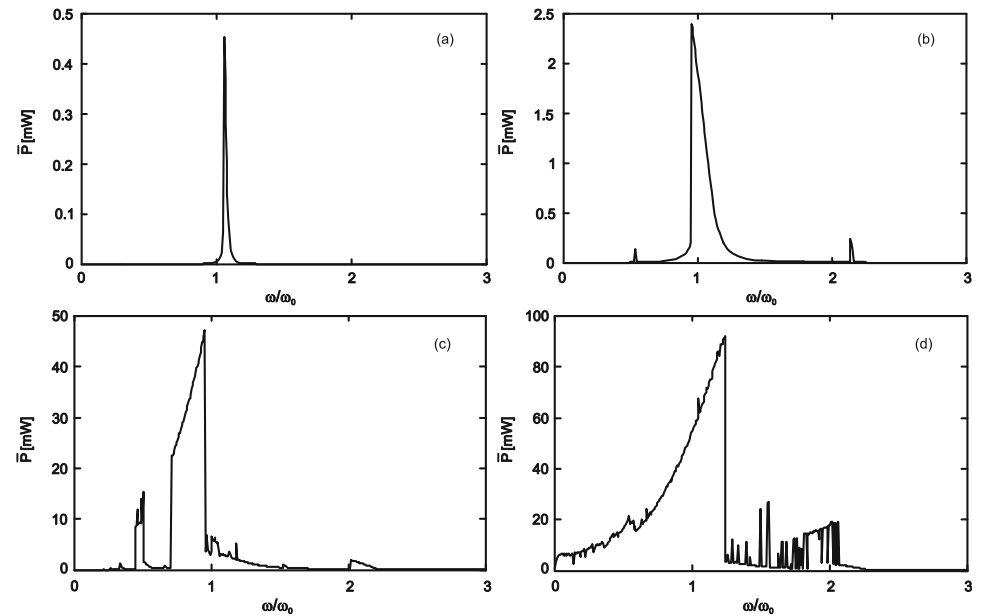

Fig. 7. Electric power vs. frequency of excitations in the nonlinear case for different values of base acceleration amplitude: (a) $A_{0}=0.1 g$; (b) $A_{0}=1 g$; (c) $A_{0}=5 g$; (d) $A_{0}=10 g$.

The electric power vs. frequency calculated in the nonlinear case at base acceleration amplitudes and frequencies similar to the linear case are shown in Fig. 7.

The excitation frequency considered in Fig. 7 is normalized, as in the linear case, to the device's natural frequency (without external magnets). This shifts the resonance peak presented in Fig. 7a towards higher frequency values. The shift is due to new stable minimum in the potential energy of the moving magnet (see inset in Fig. 5). The additional force due to the magnets causes the output power for small values of excitation force to be smaller as compared to the linear case.

On the other side we can observe (see Fig. 7) the appearing of two additional peaks in the power spectrum near the frequency values $\omega=0.5 \omega_{0}$ and $\omega=2 \omega_{0}$. At higher values of base acceleration amplitude there is large increase of the frequency range, where the harvested electrical energy is relatively high.

To compare the results in both, linear and nonlinear case, and additionally to take into account the influence of frequency spectrum width on generated power, we 


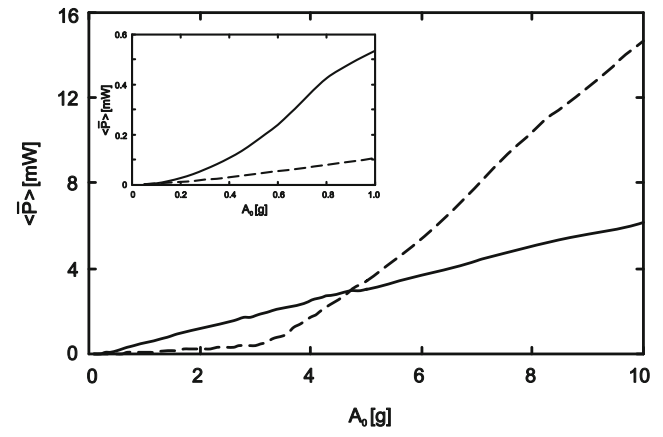

Fig. 8. The effective output power vs. base acceleration amplitude in the linear (solid line) and nonlinear (dashed line) case.
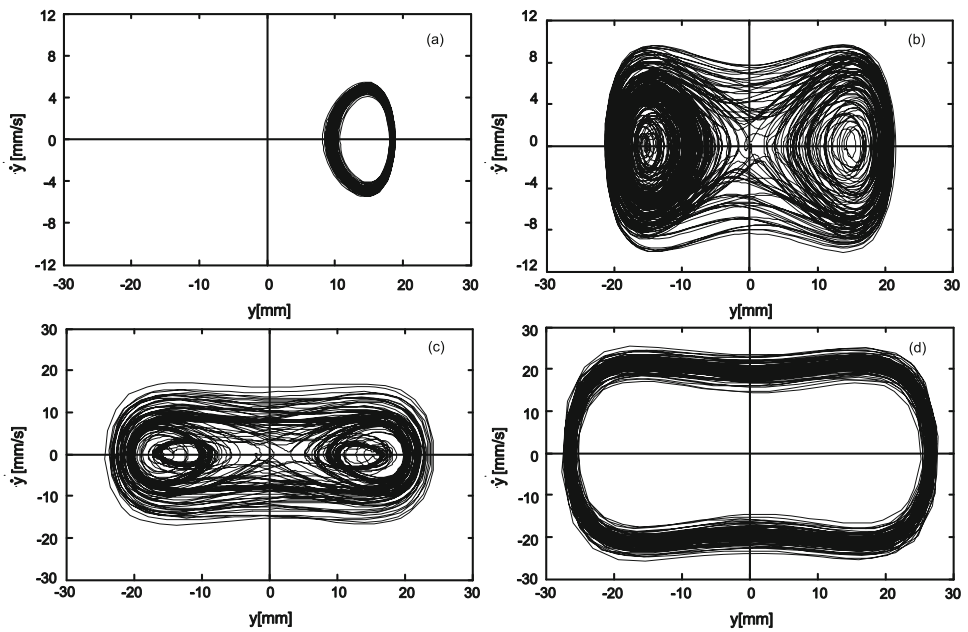

Fig. 9. Phase diagrams for the moving magnet in the nonlinear case at the resonance frequency corresponding to $A_{0}=0.1 g$ : (a) $A_{0}=2.5 g$; (b) $A_{0}=3.2 g$; (c) $A_{0}=6 g$; (d) $A_{0}=6.5 \mathrm{~g}$.

introduce the effective output power $\langle\bar{P}\rangle$, i.e. the mean value of power produced by the harvesting device, integrated over frequency range changing from 0 to $3 \omega_{0}$. The value $3 \omega_{0}$ of maximum frequency can be interpreted as the cut-off frequency, above which the generated power is negligible. In Fig. 8 we present the effective output power as a function of base acceleration amplitude in the linear and nonlinear case.

As we can see in Fig. 8 there is a significant change of the curve behavior for the nonlinear system at the base acceleration amplitude of the order of $A_{0}=3 g$. For these values of base acceleration the moving magnet starts to cross over the potential barrier and the system's nonlinearity start to play significant role. This behavior can be shown on the phase diagrams presented in Fig. 9.

In Fig. 9 we present the phase diagrams for different values of base acceleration at the frequency $\omega=1.055 \omega_{0}$ which corresponds to the system's resonance frequency for $A_{0}=0.1 \mathrm{~g}$. Our aim is to show the behavior of the system at one constant frequency versus different base accelerations. As we can see at accelerations below the $2.5 \mathrm{~g}$ the moving magnet oscillates around the local potential minimum position. When the base acceleration reaches the value of the order of $3.2 g$ the system enters the nonlinear behavior, and the magnet passes through the potential barrier at $y=0 \mathrm{~mm}$. Figure $9 \mathrm{c}$ shows that at $A_{0}=6 \mathrm{~g}$ the base acceleration amplitude is sufficiently high 

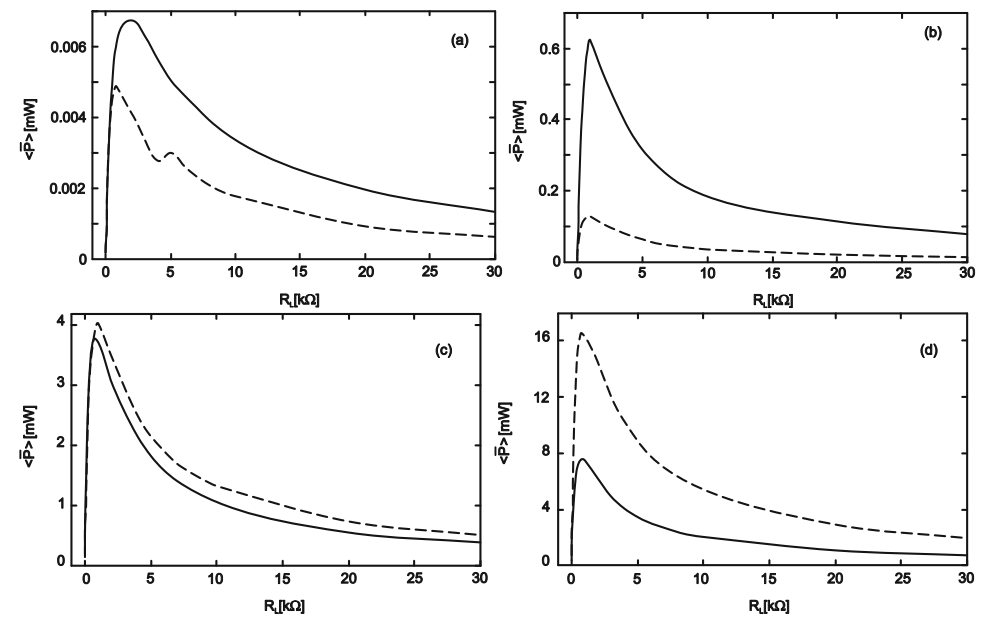

Fig. 10. The effective output power vs. electric load for different values of base acceleration amplitude (solid line - the linear case; dashed line - the nonlinear case): (a) $A_{0}=0.1 \mathrm{~g}$; (b) $A_{0}=1 g$; (c) $A_{0}=5 g$; (d) $A_{0}=10 g$.

to cause the moving magnet to start oscillating in the whole range of available displacements. If the base acceleration grows above $6.5 \mathrm{~g}$ the two-minima potential shape starts to be "invisible" for the moving magnet.

We also performe computations to examine the influence of external load on the effective power generated by the harvesting device. The results are presented in Fig. 10.

As we can see there is a typical shape of impedance matching. The data presented in Fig. 10 allows us also to obtain the value of external load when the maximum power is achieved (see e.g. [16]). Additionally, comparing the data presented in Fig. 8, we can see that the device works efficiently when the base acceleration amplitudes exceed $5 g$ 's.

All the computations in this work were done with the Mathematica and InfolyticaMagNet packages. We used the NDSolve numerical differential equation solver. The time simulation to reach the self consistent solution was of the order of one minute when the maximum number of steps to obtain the solution of coupled differential equations was set to one billion. All numerical integrations were obtained using the Monte Carlo method with the number of steps in the order of one hundred million.

\section{Conclusions}

We have examined the efficiency of energy harvesting device attached to a vibrating source that generates the harmonic excitations. The system's bistability was achieved by application of the magnetic field and the restoring force due to the springs. We have analyzed the effective mean power generated on the resistance $R_{L}$ in function of excitation frequency. The results show the broadening of the frequency spectrum where the power is relatively high in the nonlinear case (compared to the linear one).

In order to compare both systems (linear and nonlinear) we also integrated the mean power over the frequency range changing from 0 to $3 \omega_{0}$ (the effective electric power) obtaining diagrams showing the influence of excitation force amplitude on generated effective power in the linear and nonlinear case. When the base acceleration reaches some value the nonlinear system starts oscillating in the vicinity of three 
resonance frequencies around the local minima and with the higher rise of acceleration it oscillates through the potential barrier, which appears in the equilibrium position for the linear case. As result, the output electric output power exceeds the power harvested from the linear system.

This work was done due to partial support from Centre for Innovation and Transfer of Natural Sciences and Engineering Knowledge at the University of Rzeszów.

\section{References}

1. F. Cottone, H. Vocca, L. Gammaitoni, Phys. Rev. Lett. 102, 080601 (2009)

2. G. Litak, M.I. Friswell, C.A. Kitio Kwuimy, S. Adhikari, M. Borowiec, Theo. Appl. Mech. Lett. 2, 043009 (2012)

3. G. Litak, M. Borowiec, M.I. Friswell, S. Adhikari, J. Theoret. Appl. Mech. 49, 757 (2011)

4. M. Lallart, S. Pruvost, D. Guyomar, Phys. Lett. A 375, 3921 (2011)

5. A. Erturk, J. Hoffmann, D.J. Inman, Appl. Phys. Lett. 94, 254102 (2009)

6. S.C. Stanton, C.C. McGehee, B.P. Mann, Physica D 239, 640 (2010)

7. S.J. Jang, E. Rustighi, M.J. Brennan, Y.P. Lee, H.J. Jung, J. Intell. Mater. Syst. Struct. 22, 443 (2011)

8. H. Vocca, I. Neri, F. Travasso, L. Gammaitoni, Appl. Ener. 97, 771776 (2012)

9. K. Kucab, G. Górski, J. Mizia, Eur. Phys. J. Special Topics 222, 1607 (2013)

10. B.P. Mann, B.A. Owens, J. Sound Vibr. 329, 1215 (2010)

11. R. Gherca, R. Olaru, Annals University of Craiova, Electrical Engineering series, No. 35 (2011)

12. J.A.T. Machado, D. Baleanu, A.C.J. Luo (eds.), Discontinuity and Complexity in Nonlinear Physical Systems, 17 (Springer, New York, 2014)

13. Ch. Lee, D. Stamp, N.R. Kapania, J.O. Mur-Miranda, Proc. SPIE 7683, 76830Y (2010)

14. B.P. Mann, N.D. Sims, J. Sound Vibr. 319, 515 (2009)

15. D. Vokoun, M. Beleggia, L. Heller, P. Šittner, J. Magn. Magn. Mater. 321, 3758 (2009)

16. D. Spreemann, Y. Manoli, Electromagnetic Vibration Energy Harvesting Devices; Architectures, Design, Modeling and Optimization (Springer, New York, 2012) 